\title{
Simulation of three dimensional double-diffusive throughflow in internally heated anisotropic porous media
}

\begin{abstract}
A model for double-diffusive convection in an anisotropic porous layer with a constant throughflow is explored, with penetrative convection being simulated via an internal heat source. The validity of both the linear instability and global nonlinear energy stability thresholds are tested using three dimensional simulation. Our results show that the linear threshold accurately predicts on the onset of instability in the steady state throughflow. However, the required time to arrive at the steady state increases significantly as the Rayleigh number tends to the linear threshold.
\end{abstract}

Keywords: Double-diffusive convection, Throughflow, Internal heat source, Finite differences, Anisotropic porous media

\section{Nomenclature}

$\begin{array}{ll}\left(x_{1}, x_{2}, x_{3}\right)=(x, y, z) & \text { Cartesian coordinates } \\ \mathbf{v} & \text { velocity } \\ P & \text { pressure } \\ T & \text { temperature } \\ C & \text { concentration of salt } \\ \mathbf{u} & \text { dimensionless velocity } \\ p & \text { dimensionless pressure } \\ \theta & \text { dimensionless temperature } \\ \phi & \text { dimensionless concentration of salt } \\ \mu & \text { viscosity } \\ \varepsilon & \text { porosity } \\ g & \text { gravitational acceleration } \\ \kappa_{c} & \text { salt diffusivity }\end{array}$

Preprint submitted to International Journal of Heat and Mass Transfer January 6, 2014 


$\begin{array}{ll}\rho & \text { density } \\ \rho_{0} & \text { reference density } \\ T_{0} & \text { reference temperature } \\ C_{0} & \text { reference concentration } \\ \alpha_{t} & \text { thermal expansion coefficient } \\ \alpha_{c} & \text { solutal expansion coefficient } \\ K(z)=K_{0} s(z) & \text { permeability of the porous medium } \\ K_{0} & \text { reference permeability } \\ \kappa_{t} & \text { effective thermal diffusivity of the porous medium } \\ \kappa_{s} & \text { thermal diffusivity of the solid component of the porous medium } \\ \kappa_{f} & \text { thermal diffusivity of the fluid component of the porous medium } \\ c_{p} & \text { specific heat of the fluid at constant pressure } \\ c & \text { specific heat of the solid at constant pressure } \\ M & \text { ratio of heat capacities } \\ Q(>0) & \text { internal heat source } \\ R a_{L}=R_{t}^{2} & \text { thermal Rayleigh number } \\ R_{c}^{2} & \text { solute Rayleigh number } \\ T_{f} & \text { dimensionless form of the throughflow } \\ a^{2} & \text { horizontal wavenumber, } m^{2}+n^{2} \\ m, n & \text { dimensionless disturbance wave vector } \\ \vec{\omega}=\left(\xi_{1}, \xi_{2}, \xi_{3}\right) & \text { vorticity vector } \\ \vec{\psi}=\left(\psi_{1}, \psi_{2}, \psi_{3}\right) & \text { potential vector } \\ L x & \text { box dimension in the } x \text { direction } \\ L y & \text { box dimension in the } y \text { direction } \\ & \end{array}$

\section{Introduction}

Double-diffusive flows in porous media are widely encountered both in nature and in technological processes $[1,2]$. Bioremediation, where microorganisms are introduced to change the chemical composition of contaminants is a very topical area, cf. Chen et al. [3], Suchomel et al. [4]. Contaminant/pollution transport is yet another area of multi-component flow in porous media which is of much interest in environmental engineering, cf. Curran and Allen [5], Ewing and Weekes [6], Franchi and Straughan [7]. Other very important and topical areas of salt/heat transport in porous flows are in oil reservoir simulation, e.g. Ludvigsen et al. [8], and salinization in desert-like areas, Gilman and Bear [9]. 
The literature on the study of the effect of vertical throughflow on convective instability in a porous medium is much less widespread, although recent studies include Shivakumara and Suma [10], Shivakumara and Khalili [12], Shivakumara and Sureshkumar [13], Nield and Kuznetsov [14], Hill [15], Hill et al. [16] and Capone et al. [17].

The effect of vertical throughflow on double-diffusive convection in a porous medium is important due to its applications in engineering (e.g. the directional solidification of concentrated alloys as well as in some energy storage devices) and geophysics (e.g. seabed hydrodynamics such as in hydrothermal vent systems). The difficulty in dealing with such instability problems is that one has to solve time dependent equations with variable coefficients, and the work in this direction is very limited. Shivakumara and Nanjundappa [18] used linear stability theory to analytically investigate the effects of quadratic drag and vertical throughflow on double diffusive convection in a horizontal porous layer using the Forchheimer-extended Darcy equation. Shivakumara and Sureshkumar [19] investigated the effects of quadratic drag and vertical throughflow on the linear stability of a doubly diffusive Oldroyd-B-fluid-saturated horizontal porous layer. Altawallbeh et al. [20] analytically studied using both linear and weakly nonlinear stability analyses the double-diffusive convection in an anisotropic porous layer heated and salted from below with an internal heat source and Soret effect. Shivakumara and Khalili [11] studied the problem of double-diffusive convection in a fluid filled anisotropic porous layer. Hill et al. [21] studied this problem but with the presence of an internal heat source to allow penetrative convection to occur. In this paper, we explore the model presented in Hill et al. [21] of double-diffusive throughflow in an internally heated anisotropic porous medium.

When the difference between the linear (which predicts instability) and nonlinear (which predicts stability) thresholds is very large, the validity of the linear instability threshold to capture the onset of the instability is unclear. Thus, we utilise the stability analysis of Hill et al. [21] to select regions of large subcritical instabiltiies and then develop a three dimensional simulation for the problem to test the validity of these thresholds. To achieve this we transform the problem into a velocity-vorticity formulation and utilise second order finite difference schemes. We use both implicit and explicit schemes to enforce the free divergence equation.

Standard indicial notation is used throughout the article, where $\left(x_{1}, x_{2}, x_{3}\right)=$ $(x, y, z)$. 


\section{Mathematical formulation and governing equations}

Utilising the approach of Hill et al. [21] (schematically shown in Figure 1) let us consider a layer $\Omega$ of a water saturated porous medium bounded by two horizontal planes. Let $d>0, \Omega=\mathbb{R}^{2} \times(0, d)$ and $O x y z$ be a cartesian frame of reference with unit vectors $\mathbf{i}, \mathbf{i}, \mathbf{k}$.

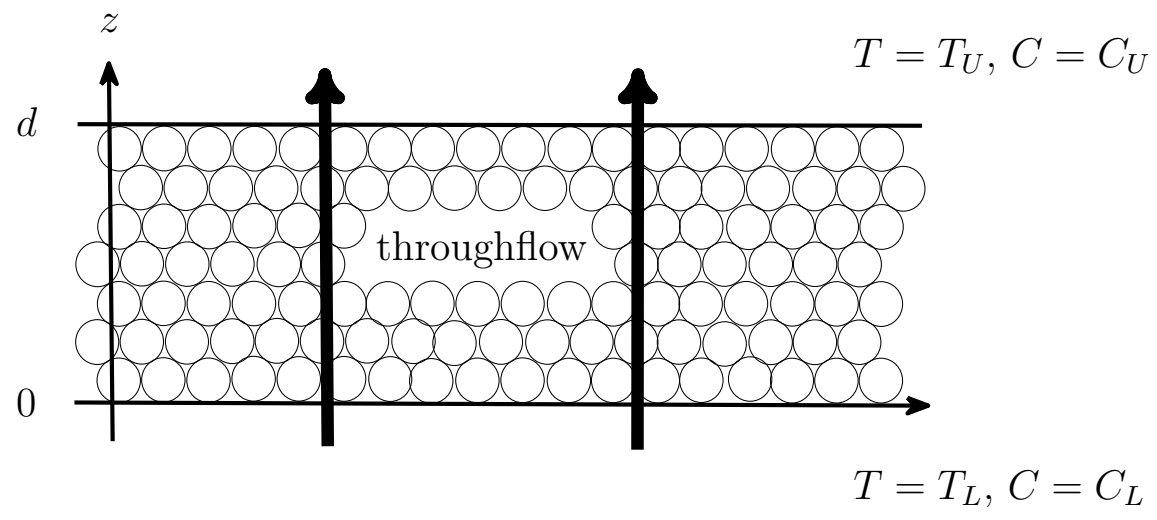

Figure 1: Schematic representation of a cross-section of the system.

Assuming that the Oberbeck-Boussinesq approximation is valid (cf. [22] and references therein), the flow in the porous medium is governed by Darcy's law

$$
\begin{gathered}
\frac{\mu}{K(z)} v_{i}=-P_{, i}-k_{i} g \rho(T, C), \\
v_{i, i}=0, \\
\frac{1}{M} T_{, t}+v_{i} T_{, i}=\kappa_{t} \nabla^{2} T+Q, \\
\varepsilon C_{, t}+v_{i} C_{, i}=\kappa_{c} \nabla^{2} C,
\end{gathered}
$$

where (2) is the incompressibility condition and (3) and (4) are the equations of energy and solute balance, respectively. The derivation of equations (1) (4) may be found in [23]. 
We have denoted $\mathbf{v}, P, T, C, \mu, \varepsilon, g$ and $\kappa_{c}$ to be the velocity, pressure, temperature, concentration of salt, viscosity, porosity, gravitational acceleration and salt diffusivity, respectively. The density $\rho$ is of the form

$$
\rho(T, C)=\rho_{0}\left(1-\alpha_{t}\left(T-T_{0}\right)+\alpha_{c}\left(C-C_{0}\right)\right)
$$

where $\rho_{0}, T_{0}$ and $C_{0}$ are a reference density, temperature and concentration, respectively, and $\alpha_{t}$ and $\alpha_{c}$ are the coefficients for thermal and solutal expansion, respectively.

The permeability of the porous medium is taken to be of the form

$$
K(z)=K_{0} s(z),
$$

where $K_{0}$ is a reference permeability and $s(z)=1+\lambda_{1} z / d$, with constant $\lambda_{1}>$ -1 to ensure $s(z)>0$. The effective thermal conductivity of the saturated porous medium $\kappa_{t}$ is defined by the ratio between the thermal diffusivity of the porous medium and the heat capacity per unit volume of the fluid:

$$
\kappa_{t}=\frac{(1-\varepsilon) \kappa_{s}+\varepsilon \kappa_{f}}{\left(\rho_{0} c_{p}\right)_{f}}
$$

where $\kappa_{s}$ and $\kappa_{f}$ are the thermal diffusivities of the solid and fluid components of the porous medium, respectively and $c_{p}$ is the specific heat of the fluid at constant pressure. The coefficient $M$ is the ratio of heat capacities defined by

$$
M=\frac{\left(\rho_{0} c_{p}\right)_{f}}{\left(\rho_{0} c\right)_{m}} .
$$

In (5) $c$ is the specific heat of the solid, and

$$
\left(\rho_{0} c\right)_{m}=(1-\varepsilon)\left(\rho_{0} c\right)_{s}+\varepsilon\left(\rho_{0} c_{p}\right)_{f},
$$

denotes the overall heat capacity per unit volume of the porous medium. The subscripts $f, s$ and $m$ referring to the fluid, solid and porous components of the medium, respectively.

The $Q(>0)$ term in (3) is a (constant) internal heat source, with its inclusion allowing the model to describe penetrative convection in the porous layer [24].

The temperature and concentration boundary conditions for the problem are $T=T_{U}$ and $C=C_{U}$ at $z=d$ and $T=T_{L}$ and $C=C_{L}$ at $z=0$, where 
$C_{L}>C_{U}$, so that the system is being salted from below. We allow for the two cases of heating from below $T_{L}>T_{U}$ and from above $T_{L}<T_{U}$.

Let us now consider the basic steady state solution of $(1)-(4)$, with a throughflow in the $z$ direction of the form

$$
\overline{\mathbf{v}}=(0,0, V),
$$

where $V$ is constant. Utilising the boundary conditions, Eqs. (3) and (4) yield the temperature and concentration steady states

$$
\begin{gathered}
\bar{T}(z)=\frac{Q z}{V}+T_{L}+\frac{V\left(T_{L}-T_{U}\right)+Q d}{V\left(e^{\frac{V d}{\kappa_{t}}}-1\right)}\left(1-e^{\frac{V z}{\kappa_{t}}}\right), \\
\bar{C}(z)=C_{L}+\frac{C_{L}-C_{U}}{1-e^{\frac{V d}{\kappa_{c}}}}\left(e^{\frac{V z}{\kappa_{c}}}-1\right) .
\end{gathered}
$$

To investigate the stability of these solutions, we introduce perturbations $\left(u_{i}, p, \theta, \phi\right)$ by

$$
v_{i}=u_{i}+\bar{v}_{i}, \quad P=p+\bar{P}, \quad T=\theta+\bar{T}, \quad C=\phi+\bar{C} .
$$

The perturbation equations are nondimensionalized according to the scales (stars denote dimensionless quantities)

$$
\begin{gathered}
p=\frac{\mu \kappa_{t}}{K_{0}} p^{*}, \quad \theta=\theta^{*} \sqrt{\frac{d Q \mu}{g \rho_{0} \alpha_{t} K_{0}}}, \quad x_{i}=d x_{i}^{*}, \quad \phi=\phi^{*} \sqrt{\frac{\mu \kappa_{t}\left(C_{L}-C_{U}\right)}{g \rho_{0} \alpha_{c} K_{0} d}}, \\
u_{i}=\frac{\kappa_{t}}{d} u_{i}^{*}, \quad t=\frac{d^{2}}{\kappa_{t} M} t^{*}, \quad \widehat{\varepsilon}=M \varepsilon, \quad L e=\frac{\kappa_{t}}{\kappa_{c}}, \quad T_{f}=\frac{V d}{\kappa_{t}}, \\
R_{t}^{2}=\frac{g \rho_{0} \alpha_{t} K_{0} d^{3} Q}{\mu \kappa_{t}^{2}}, \quad R_{c}^{2}=\frac{g \rho_{0} \alpha_{c} K_{0} d\left(C_{L}-C_{U}\right)}{\mu \kappa_{t}}, \quad \epsilon=\frac{\left(T_{L}-T_{U}\right) \kappa_{t}}{Q d^{2}}
\end{gathered}
$$

where $R_{t}^{2}$ and $R_{c}^{2}$ are the thermal and solute Rayleigh numbers, respectively, and $T_{f}$ is the non-dimensional form of the throughflow. The dimensionless perturbation equations are (after omitting all stars)

$$
\begin{gathered}
\frac{1}{f(z)} u_{i}=-p_{, i}+R_{t} \theta k_{i}-R_{c} \phi k_{i}, \\
u_{i, i}=0,
\end{gathered}
$$




$$
\begin{gathered}
\theta_{, t}+u_{i} \theta_{, i}+T_{f} \theta_{, 3}=R_{t} f_{1}(z) w+\nabla^{2} \theta, \\
\widehat{\varepsilon} \phi_{, t}+u_{i} \phi_{, i}+T_{f} \phi_{, 3}=R_{c} f_{2}(z) w+\frac{1}{L e} \nabla^{2} \phi,
\end{gathered}
$$

with $w=u_{3}$ and $f(z)=1+\lambda_{1} z$ (with $\lambda_{1}>-1$ to ensure $f(z)>0$ ),

$$
\begin{gathered}
f_{1}(z)=\frac{T_{f}}{e^{T_{f}}-1}\left(\epsilon+\frac{1}{T_{f}}\right) e^{T_{f} z}-\frac{1}{T_{f}}, \\
f_{2}(z)=\frac{L e T_{f} e^{L e T_{f} z}}{e^{L e T_{f}}-1} .
\end{gathered}
$$

It is important to note that $\epsilon>0$ and $\epsilon<0$ correspond to heating from below and above, respectively. These equations hold in the region $\{z \in$ $(0,1)\} \times\left\{(x, y) \in \mathbb{R}^{2}\right\}$ and the boundary conditions to be satisfied are:

$$
\mathbf{u}=0, \quad \theta=0, \quad \phi=0, \quad \text { at } \quad z=0,1,
$$

where $u_{i}, p, \theta$ and $\phi$ are assumed periodic in the $x$ and $y$ directions.

\section{Linear and nonlinear energy stability theories}

Linear instability results for stationary convection are obtained via the application of standard procedures to the linearized version of Eqs. (6)(9). Following the approach of Hill et al. [21] the critical linear instability thresholds are located through the following eigenvalue problem for growth rate $\sigma$

$$
\begin{gathered}
f\left(D^{2}-a^{2}\right) W-D f D W+a^{2} R_{t} f^{2} \Theta-a^{2} R_{c} f^{2} \phi=0, \\
\left(D^{2}-\zeta a^{2}\right) \Theta-T_{f} D \Theta+R_{t} f_{1}(z) W=\sigma \Theta, \\
\frac{1}{L e}\left(D^{2}-a^{2}\right) \phi-T_{f} D \phi+R_{c} f_{2}(z) W=\widehat{\varepsilon} \sigma \phi,
\end{gathered}
$$

on $z \in(0,1)$. Here $D=d / d z, w=W e^{i(m x+n y)}, \theta=\Theta e^{i(m x+n y)}, \phi=$ $\Phi e^{i(m x+n y)}$ and $a^{2}=m^{2}+n^{2}$ is a horizontal wavenumber. These equations arc subject to the boundary conditions

$$
W=\Theta=\Phi=0, \quad \text { at } z=0,1 .
$$

Linearized instability theory locates where instability occurs. It does not, however, a priori yield any information on stability, nor does it necessarily 
predict the smallest instability threshold. It is possible that nonlinear terms will make a system become unstable long before the threshold predicted by linear theory is reached. Such instabilities are called subcritical. If we have a threshold below which we know all nonlinear perturbations decay, in a precise mathematical way, then this will yield a nonlinear stability boundary. When this threshold is relatively close to the analogous threshold of linear theory we can conclude that the linear results are actually predicting the physical picture correctly.

Hill et al. [21] developed an unconditional nonlinear energy stability theory for system (6)-(9) with the following eigenvalue problem:

$$
\begin{gathered}
f\left(D^{2}-a^{2}\right) W-D f D W+a^{2} R_{t} f^{2}(z) \Theta-a^{2} R_{c} f^{2}(z) \Phi=0, \\
2\left(D^{2}-a^{2}\right) \Theta+R_{t} f_{1}(z) W+a^{2} R f(z) \Psi=0, \\
\frac{2 \lambda}{L e}\left(D^{2}-a^{2}\right) \Phi+R_{c} \lambda f_{2}(z) W-a^{2} R_{c} f(z) \Psi=0, \\
f^{2}\left(D^{2}-a^{2}\right) \Psi+f D f D \Psi+f \Psi D^{2} f-(D f)^{2} \Psi+R_{t} f^{2}(z) f_{1}(z) \Theta \\
+\lambda R_{c} f^{2}(z) f_{2}(z) \Phi=0,
\end{gathered}
$$

with the boundary conditions

$$
W=\Theta=\Phi=\Psi=0, \quad \text { at } z=0,1,
$$

where $\lambda$ is a parameter to be chosen and $\Phi$ is the normal mode representation of Lagrange multiplier (for more detail see [21]). The critical eigenvalue $R_{E}\left(a^{2} ; \lambda\right)$ can be found from

$$
R a_{E}=\max _{\lambda} \min _{a^{2}} R_{t}^{2}\left(a^{2} ; \lambda\right)
$$

We solve the eigenvalue systems (11)-(13) for $\sigma$ and (15)-(18) for $R_{t}$ numerically using three different numerical techniques to ensure accuracy, namely Chebyshev collocation [25], finite elements [26] and finite differences methods cf. [27]. The results are discussed in Section 6.

\section{Velocity-vorticity formulation}

In Sections 4 and 5 we now develop a three-dimensional approach to solve the time dependent governing equations (1) - (4) in order to assess 
the accuracy of the linear instability and nonlinear stability thresholds. A schematic diagram of the three-dimensional space (based on Figure 1) under consideration is given in Section 6.

Fasel [28] was the first article to address the velocity-vorticity form of the Navier-Stokes equations, and established this formula as an effective formulation for the solution of incompressible viscous flow problems. The velocity-vorticity form of the momentum equations is one of the best choices to achieve the divergence-free velocity field constraint for the incompressible Navier-Stokes equations in three dimensions. Moreover, the natural convection problem can be simulated directly by the velocity-vorticity formulation, without the need to handle the pressure term. For the case of incompressible fluid flows, if the incompressibility condition imposed by the continuity equation is satisfied by some means, then a divergence-free flow field can be computed by solving the velocity-vorticity equations cf. Fasel [28], Napolitano and Catalano [29], Guj and Stella [30], Davis and Carpenter [31], Wong and Baker [32].

Mallinson and de Vahl Davis [33] first explored two dimensional natural convection in a rectangular box, with the proposition of the vorticity-stream function formulation without the pressure term. The problem of natural convection in a cubic enclosure has been studied using the velocity-vorticity equations by Wong and Baker [32]. However, there are two issues related with the velocity-vorticity formulation. Firstly, the number of variables is increased from four to six as compared to the primitive-variable form for three-dimensional problems. Secondly, it is not easy to enforce the vorticity definition at the solid boundaries to satisfy the continuity equation [34]. Davis and Carpenter [31] introduced a very important solution to the first problem, where only three governing equations were solved by considering two velocities and one vorticity as the primitive variables and computed the remaining three field variables as secondary variables. They handled the convective part of the governing equations using a predictor-corrector scheme, thus not deviating substantially from the existing algorithms for treating the pressure term. The solution of the second problem requires a higher-order scheme to compute the boundary vorticity values. Wong and Baker [32] introduced a second-order accurate Taylor's series expansion to compute the vorticity values at the boundaries. Davis and Carpenter [31] used an integral approach for vorticity definition at the boundary, as followed by Guevremont et al. [35].

In this paper, we present an efficient, stable, and accurate finite dif- 
ference schemes in the vorticity-vector potential formulation for computing the convective motion of an incompressible fluid in a porous material. The emphasis is on three dimensions and nonstaggered grids. We introduce a second-order accurate method based on the vorticity-vector potential formulation on the nonstaggered grid whose performance on uniform grids is comparable with the finite scheme. We will pay special attention to how accurately the divergence-free conditions for vorticity, velocity, and vector potential are satisfied. We will derive the three-dimensional analog of the local vorticity boundary conditions.

By using the curl operator to Eq. (6), one gets the following dimensionless form of the vorticity transport equation:

$$
\frac{1}{f(z)} \vec{\omega}-\frac{f^{\prime}(z)}{f^{2}(z)}(-v, u, 0)=R \nabla \times \theta \mathbf{k}-R_{c} \nabla \times \phi \mathbf{k},
$$

where the vorticity vector $\vec{\omega}=\left(\xi_{1}, \xi_{2}, \xi_{3}\right)$ is defined as

$$
\vec{\omega}=\nabla \times \vec{v} \text {. }
$$

To calculate velocity from vorticity, it is convenient to introduce a vector potential $\vec{\psi}=\left(\psi_{1}, \psi_{2}, \psi_{3}\right)$, which may be looked upon as the three-dimensional counterpart of two-dimensional stream function. The vector potential is defined by

$$
\vec{v}=\nabla \times \vec{\psi}
$$

It easy to show the existence of such a vector potential for a solenoidal vector field $(\nabla \cdot \vec{v}=0)$, which is required to be solenoidal, i.e.,

$$
\nabla \cdot \vec{\psi}=0
$$

Substituting Eq. (22) in Eq. (21) and using Eq. (23) yields

$$
\nabla^{2} \vec{\psi}=-\vec{\omega} \text {. }
$$

The set of equations (8), (9), (20), (22) and (24) with appropriate boundary conditions form the basis for the numerical computations. The boundary conditions for the vector potential are given below

$$
\frac{\partial \psi_{1}}{\partial x}=\psi_{2}=\psi_{3}=0, \quad \text { at } \quad x=0,1
$$




$$
\begin{aligned}
& \psi_{1}=\frac{\partial \psi_{2}}{\partial y}=\psi_{3}=0, \quad \text { at } \quad y=0,1 \\
& \psi_{1}=\psi_{2}=\frac{\partial \psi_{3}}{\partial z}=0, \quad \text { at } \quad z=0,1 .
\end{aligned}
$$

The boundary conditions on vorticity follow directly and may expressed as

$$
\begin{aligned}
& \xi_{1}=0, \xi_{2}=-\frac{\partial w}{\partial x}, \xi_{3}=\frac{\partial v}{\partial x}, \quad \text { at } \quad x=0,1, \\
& \xi_{1}=\frac{\partial w}{\partial y}, \xi_{2}=0, \xi_{3}=-\frac{\partial u}{\partial y}, \quad \text { at } \quad y=0,1, \\
& \xi_{1}=-\frac{\partial v}{\partial z}, \xi_{2}=\frac{\partial u}{\partial z}, \xi_{3}=0, \quad \text { at } z=0,1 .
\end{aligned}
$$

\section{Numerical schemes}

The first step in the numerical computational is to give an initial values for the vorticity vectors $\xi_{1 i j k}^{n}, \xi_{2 i j k}^{n}, \xi_{3 i j k}^{n}, i, j, k=0,1, \ldots, m$. Next, the Poisson equation (24) is discretized in space using an implicit scheme as follows

$$
\begin{aligned}
& \left(\delta_{x}^{2}+\delta_{y}^{2}+\delta_{z}^{2}\right) \psi_{1 i j k}^{n+1}=-\xi_{1 i j k}^{n}, \\
& \left(\delta_{x}^{2}+\delta_{y}^{2}+\delta_{z}^{2}\right) \psi_{2 i j k}^{n+1}=-\xi_{2 i j k}^{n}, \\
& \left(\delta_{x}^{2}+\delta_{y}^{2}+\delta_{z}^{2}\right) \psi_{3 i j k}^{n+1}=-\xi_{3 i j k}^{n},
\end{aligned}
$$

where $\delta_{x}^{2}, \delta_{y}^{2}, \delta_{z}^{2}$ are the second-order central difference operators, which are defined as

$$
\begin{aligned}
\delta_{x}^{2} \phi & =\frac{\phi_{i+1 j k}-2 \phi_{i j k}+\phi_{i-1 j k}}{(\Delta x)^{2}}, \\
\delta_{y}^{2} \phi & =\frac{\phi_{i j k+1}-2 \phi_{i j k}+\phi_{i j-1 k}}{(\Delta y)^{2}}, \\
\delta_{z}^{2} \phi & =\frac{\phi_{i j k+1}-2 \phi_{i j k}+\phi_{i j k-1}}{(\Delta z)^{2}} .
\end{aligned}
$$

The Gauss-Seidel iteration method is utilised to evaluate $\psi_{1 i j k}^{n+1}, \psi_{2 i j k}^{n+1}, \psi_{3 i j k}^{n+1}$, $i, j, k=1, \ldots, m-1$ from Eqs. (31), (32), (33), respectively. The next step 
is to discretize Eqs.(25)-(27) to evaluate the $\psi_{10 j k}^{n+1}, \psi_{1 m j k}^{n+1}, \psi_{2 i 0 k}^{n+1}, \psi_{2 i m k}^{n+1}, \psi_{3 i j 0}^{n+1}$, $\psi_{3 i j m}^{n+1}, i, j, k=0, \ldots, m$ i.e. we used Eqs. (25)-(27) to evaluate the potential vectors at the boundary. Now, the velocity vector can be calculated explicitly by using a second order finite difference scheme to Eq. (22) as follows:

$$
\begin{gathered}
u_{i j k}^{n+1}=\delta_{y} \psi_{3 i j k}^{n+1}-\delta_{z} \psi_{2 i j k}^{n+1}, \\
v_{i j k}^{n+1}=\delta_{z} \psi_{1 i j k}^{n+1}-\delta_{x} \psi_{3 i j k}^{n+1}, \\
u_{i j k}^{n+1}=\delta_{x} \psi_{2 i j k}^{n+1}-\delta_{y} \psi_{1 i j k}^{n+1}, \\
i, j, k=1, \ldots, m-1,
\end{gathered}
$$

where $\delta_{x}, \delta_{y}, \delta_{z}$ are the first-order central difference operators, which are defined as

$$
\begin{aligned}
\delta_{x} \phi & =\frac{\phi_{i+1 j k}-\phi_{i-1 j k}}{2 \Delta x}, \\
\delta_{y} \phi & =\frac{\phi_{i j+1 k}-\phi_{i j-1 k}}{2 \Delta y}, \\
\delta_{z} \phi & =\frac{\phi_{i j k+1}-\phi_{i j k-1}}{2 \Delta z} .
\end{aligned}
$$

The vorticity transport equation (20) is discretized in time using the explicit scheme. The discretized form of the vorticity transport equations (20) for the three vorticity components and energy equations (8) and (9) can be written as

$$
\begin{gathered}
\frac{1}{f_{k}} \xi_{1 i j k}^{n+1}=\frac{f_{k}^{\prime}}{f_{k}^{2}} v_{i j k}^{n+1}+R \delta_{y} \theta_{i j k}^{n+1}-R_{c} \delta_{y} \phi_{i j k}^{n+1} \\
\xi_{2 i j k}^{n+1}=-\frac{f_{k}^{\prime}}{f_{k}^{2}} u_{i j k}^{n+1}-R \delta_{x} \theta_{i j k}^{n+1}+R_{c} \delta_{x} \phi_{i j k}^{n+1} \\
\xi_{3 i j k}^{n+1}=0, \\
\frac{\theta_{i j k}^{n+1}-\theta_{i j k}^{n}}{\Delta t}+u_{i j k}^{n} \delta_{x} \theta_{i j k}^{n}+v_{i j k}^{n} \delta_{y} \theta_{i j k}^{n}+w_{i j k}^{n} \delta_{z} \theta_{i j k}^{n} \\
+T_{f} \delta_{z} \theta_{i j k}^{n}=R f_{1 k} w_{i j k}^{n}+\left(\delta_{x}^{2}+\delta_{y}^{2}+\delta_{z}^{2}\right) \theta_{i j k}^{n}, \\
\widehat{\varepsilon}\left(\frac{\phi_{i j k}^{n+1}-\phi_{i j k}^{n}}{\Delta t}\right)+u_{i j k}^{n} \delta_{x} \phi_{i j k}^{n}+v_{i j k}^{n} \delta_{y} \phi_{i j k}^{n}+w_{i j k}^{n} \delta_{z} \phi_{i j k}^{n} \\
+T_{f} \delta_{z} \phi_{i j k}^{n}=R_{c} f_{2 k} w_{i j k}^{n}+\frac{1}{L e}\left(\delta_{x}^{2}+\delta_{y}^{2}+\delta_{z}^{2}\right) \phi_{i j k}^{n},
\end{gathered}
$$




$$
i, j, k=1, \ldots, m-1 \text {. }
$$

The temperature on the boundary can be computed explicitly using Eqs. (10). However, a second order implicit technique has been used to evaluated the vorticity vector at the boundary form Eqs.(28)-(30). To enforce the vorticity definition at the wall, we used a Taylor's series expansion to compute the vorticity values at the boundaries.

Here, we should mention that our scheme is flexible for various $R a$ values and thus the grid resolution has been selected according to the $R a$ values. We decrease the values of $\Delta x, \Delta y$ and $\Delta z$ as the value of $R a$ increases. However, for double-diffusive throughflow in porous media problem, we find that $\Delta x=\Delta y=\Delta z=0.02$ is enough to give us very accurate results.

\section{Results and conclusions}

To locate the stability thresholds, eigenvalue systems (11)-(13) and (15)(18) have been solved using Chebyshev collocation, finite elements and finite differences methods.

In our use of the Chebyshev collocation method, we used between 20 and 30 polynomials. Usually 25 was found to be sufficient but convergence was checked by varying the number of polynomials and by examining the convergence of the associated eigenvector (which yields the approximate associated eigenfunction). For the finite elements method, we found that convergence to 8 decimal places is achieved with 3 elements, which each element having 11 nodes. For the finite differences scheme we found that convergence to 8 decimal places is achieved with $h=0.001$. These techniques were selected due to their flexibility and accuracy cf. [25, 26, 27].

With respect to the physical system, instability refers to the destabilization of the steady state throughflow after a perturbation. If the flow returns to the steady state throughflow after a perturbation, this is stability. In this section, $R a_{L}$, is the critical Rayleigh number for linear instability and $R a_{E}$ is the global nonlinear stability threshold.

The corresponding critical wavenumbers of the linear instability and global nonlinear stability will be denoted by $a_{L}^{2}$ and $a_{E}^{2}$. In Table 1 , we present numerical results of the linear instability and nonlinear stability analyses. The dimensions of the box, which are calculated according to the critical wavenumber, are shown in Table 1 . In this table $L x$ and $L y$ are box dimensions in the $x$ and $y$ directions, respectively. The box dimension 
in the $z$ direction is always equal to 1 . We assume that the perturbation fields $(u, \theta, \pi)$ are periodic in the $x$ and $y$ directions and denote by $\Omega=\left[0,2 \pi / a_{x}\right] \times\left[0,2 \pi / a_{y}\right] \times[0,1]$ to be the periodicity cell, where $a_{x}$ and $a_{y}$ are the wavenumbers in the $x$ and $y$ directions, respectively. $a_{x}$ and $a_{y}$ are evaluated according to the critical wavenumbers $a_{L}^{2}$ where $a_{L}^{2}=a_{x}^{2}+a_{y}^{2}$, where $L x=2 \pi / a_{x}$ and $L y=2 \pi / a_{y}$. The values of $L x$ and $L y$ in Table 1 may be rearranged to yield a number of possible solutions for each value of the critical wavenumbers. However, we select a solution so that these two values are similar to avoid any possible stabilisation effect from of walls.

We select the situations which have large subcritical regions, where the the linear threshold substantially different from the nonlinear one. This is a region of physical parameters for which the throughflow may potentially become unstable before the linear instability thresholds predicts it should.

To derive numerical solutions of the time dependent fully three dimensional problem, we use $\Delta t=5 \times 10^{-5}$ and $\Delta x=\Delta y=\Delta z=0.02$. The convergence criteria has been selected to make sure that the solutions arrive at a steady state. The convergence criteria is

$$
\varphi=\max _{i, j, k}\left\{\left|\xi_{1 i j k}^{n+1}-\xi_{1 i j k}^{n}\right|,\left|\xi_{2 i j k}^{n+1}-\xi_{2 i j k}^{n}\right|,\left|\xi_{3 i j k}^{n+1}-\xi_{3 i j k}^{n}\right|,\left|\theta_{i j k}^{n+1}-\theta_{i j k}^{n}\right|\right\},
$$

and we select $\varphi=10^{-6}$. The program will continue computing the results of the temperature, velocity, vorticity and potential vector for new time levels until the results stratify the convergence criteria, otherwise, we stop the program after 80000 time levels, i.e at the time $\tau=4$.

To solve eqs. (31) - (33) using the Gauss-Seidel iteration method, in the first time level we give an initial value to the potential vector and we denote $\psi_{1 i j k}^{1, k}, \psi_{2 i j k}^{1, k}, \psi_{3 i j k}^{1, k}$ to be the potential vector. Then, using these initial values, we compute new values which we denote by $\psi_{1 i j k}^{1, k+1}, \psi_{2 i j k}^{1, k+1}, \psi_{3 i j k}^{1, k+1}$ and use these values to evaluate new values. The program will continue in this process until the convergence criteria is satisfied, which is

$$
\eta=\max _{i, j, k}\left\{\left|\psi_{1 i j k}^{1, k+1}-\psi_{1 i j k}^{1, k}\right|,\left|\psi_{2 i j k}^{1, k+1}-\psi_{2 i j k}^{1, k}\right|,\left|\psi_{3 i j k}^{1, k+1}-\psi_{3 i j k}^{1, k}\right|\right\}<10^{-5} .
$$

In the next time levels, the values of $\psi_{1 i j k}, \psi_{2 i j k}, \psi_{3 i j k}$ in the time level $n$ will be the initial values to the next time level.

In order to display the numerical results clearly, the temperature, velocity and vorticity contours are plotted on various mid-planes along the principle axes of the box cavity with mesh size of $91 \times 61 \times 51, z=0.5, L e=5, \lambda=0.1$, 
$T_{f}=6, \epsilon=0.1, R_{c}=10, R^{2}=782, \Delta t=5 \times 10^{-5}, \Delta x=\Delta y=\Delta z=$ 0.02 . In Figure 2, the concentration, temperature and velocity contours are presented at the time level $\tau=4$ as the possibility of the solution arriving at the steady state is impossible due to the convergence criteria.

Figure 2 shows the contours of $u, v, w, \phi$ and $\theta$ in $(a),(b),(c),(d),(e)$ and $(f)$ respectively.

In Tables $2-4$, we show a summary of the numerical results where we introduce the maximum and minimum values of temperature, velocity, vorticity and potential vectors. In Table 2 , we select $T_{f}=6, \epsilon=0.1$, $L e=5, \lambda=0.1$ and $R_{c}=5$, then according to the stability analysis we have $R a_{L}=638.2956, R a_{E}=418.1652, L x=1.4$ and $L y=1.2$. Here, it clear we have very large subcritical stability region as there is a big difference between the critical Rayleigh numbers of linear and nonlinear theories. From Table 2 , for $R^{2}=588$, we can see that the values of temperature, velocity, vorticity and potential vectors satisfy the convergence criteria at $\tau=1.25715$ and thus the solution arrive to the basic steady state within a short time. However, for $R^{2}=625$, the program needs $\tau=3.34195$ to arrive to the basic steady state, which is expected as the the required time to arrive at a steady state increases with increasing $R^{2}$ values until the solution does not arrive at any steady state. Moreover, for $R^{2}=644$, the solutions do not arrive at any steady state and the program stops at $\tau=4$. For $R^{2}=644$, we let the program work run for a significant period to test the convection's long term behavior. We see that the values of the velocities increase at $\tau=8$, and then decrease at $\tau=12$ and continue in this oscillation. Here, according to the numerical results, the linear instability threshold is the actual threshold, i.e. the solutions arrive to the basic steady state before the linear instability threshold. However, the results of Tables 3 and 4 explain that the stability behavior is similar to the stability behavior of Table 2, as we found that the actual threshold is close to the linear instability threshold.

\section{Conclusions}

In this paper we have explored double-diffusive convection in an anisotropic porous layer with a constant throughflow. Regions of very large subcritical instabilities, i.e. where agreement between the linear instability thresholds and nonlinear stability thresholds is poor, are studied by solving for the full three-dimensional system. The results indicate that the linear threshold accurately predicts on the onset of instability in the basic steady state. How- 


\begin{tabular}{cccccccc}
\hline$T_{f}$ & $\epsilon$ & $R_{c}$ & $R a_{L}$ & $a_{L}^{2}$ & $R a_{E}$ & $L y$ & $L x$ \\
\hline 6 & 0.1 & 5 & 638.2956 & 47.2488 & 418.1652 & 1.4 & 1.2 \\
8 & 0.1 & 10 & 904.3747 & 58.7498 & 414.5583 & 1.4 & 1 \\
-11 & 0.5 & 5 & 318.1784 & 39.3551 & 155.5336 & 1.8 & 1.2 \\
\hline
\end{tabular}

Table 1: Critical Rayleigh and wavenumbers $R a_{L}, R a_{E}, a_{L}^{2}$ at $L e=5$ and $\lambda=0.1$.

ever, the required time to arrive at the steady state increases significantly as the Rayleigh number tends to the linear threshold.

We find that the linear instability threshold $\left(R a_{L}\right)$ gives an accurate prediction to the physical conditions under which the steady state throughflow will destabilise. If the Rayleigh number $R^{2}$ is less than $R a_{L}$, the temperature, velocity, vorticity and potential perturbations vanish, sending the solution back to the steady state, before the linear thresholds are reached. Numerically, the required time to arrive at the steady state increases as the value of $R^{2}$ increases. When $R^{2}$ is close to $R a_{L}$, the solutions can tend to a steady state which is different to the basic steady state $\bar{v}=(0,0, V)$. When $R^{2}>R a_{L}$ the steady state throughflow destabilises, with oscillating perturbations.

Finally, we can see that the stability results of the first two cases in Table 1 are different from the last one. The difference is that the position of the actual threshold. For the last case, it is really that the actual threshold is close to linear threshold but there is big difference between the actual and the linear thresholds. However, for the first two cases the actual threshold was very close to the critical Rayleigh number of linear theory. As we believe, this is because the system become more unsymmetric when $T_{f}<0$ and as the negativity value of $T_{f}$ increase the actual threshold will be closer to the critical Rayleigh number of the nonlinear theory. However, when the value of $T_{f}$ is positive the system become more symmetric and thus the actual threshold is close to the critical Rayleigh number of the linear theory. The effect of $T_{f}$ connected with natural of the functions $f_{1}$ and $f_{2}$, where these functions are defined as exponential functions and the variation of the exponential functions increase rapidly as their exponents increase. 


\begin{tabular}{ccccccc}
\hline & \multicolumn{3}{c}{$R^{2}=588$} & \multicolumn{2}{c}{$R^{2}=625$} & \multicolumn{2}{c}{$R^{2}=644$} \\
\hline & \multicolumn{2}{c}{$\tau=1.25715$} & \multicolumn{2}{c}{$\tau=3.34195$} & \multicolumn{2}{c}{$\tau=4$} \\
\hline & Max & Min & Max & Min & Max & Min \\
\hline$u$ & $4.62 \mathrm{E}-05$ & $-4.48 \mathrm{E}-05$ & $1.54 \mathrm{E}-04$ & $-1.45 \mathrm{E}-04$ & 0.5274 & -0.4678 \\
$v$ & $3.41 \mathrm{E}-05$ & $-3.40 \mathrm{E}-05$ & $1.06 \mathrm{E}-04$ & $-1.06 \mathrm{E}-04$ & 0.3233 & -0.3233 \\
$w$ & $6.87 \mathrm{E}-05$ & $-2.29 \mathrm{E}-05$ & $2.21 \mathrm{E}-04$ & $-8.73 \mathrm{E}-05$ & 0.7107 & -0.3560 \\
$\xi_{1}$ & $1.57 \mathrm{E}-03$ & $-1.56 \mathrm{E}-03$ & $4.92 \mathrm{E}-03$ & $-4.92 \mathrm{E}-03$ & 15.2392 & -15.2402 \\
$\xi_{2}$ & $2.05 \mathrm{E}-03$ & $-2.12 \mathrm{E}-03$ & $6.74 \mathrm{E}-03$ & $-7.17 \mathrm{E}-03$ & 22.0174 & -24.6786 \\
$\xi_{3}$ & $2.17 \mathrm{E}-04$ & $-2.24 \mathrm{E}-04$ & $5.87 \mathrm{E}-04$ & $-5.86 \mathrm{E}-04$ & 2.4264 & -2.4248 \\
$\psi_{1}$ & $4.40 \mathrm{E}-06$ & $-4.42 \mathrm{E}-06$ & $1.33 \mathrm{E}-05$ & $-1.33 \mathrm{E}-05$ & 0.0398 & -0.0398 \\
$\psi_{2}$ & $6.00 \mathrm{E}-06$ & $-5.84 \mathrm{E}-06$ & $1.96 \mathrm{E}-05$ & $-1.84 \mathrm{E}-05$ & 0.0661 & -0.0579 \\
$\theta$ & $6.48 \mathrm{E}-06$ & $-1.82 \mathrm{E}-06$ & $1.98 \mathrm{E}-05$ & $-6.75 \mathrm{E}-06$ & 0.0627 & -0.0290 \\
$\phi$ & $1.04 \mathrm{E}-05$ & $-3.39 \mathrm{E}-06$ & $3.16 \mathrm{E}-05$ & $-1.22 \mathrm{E}-05$ & 0.0980 & -0.0496 \\
\hline
\end{tabular}

Table 2: Summary of numerical results for $T_{f}=6, \epsilon=0.1, L e=5, \lambda=0.1, R_{c}=5$, $R a_{L}=638.2956, R a_{E}=418.1652, L x=1.4$ and $L y=1.2$.

\begin{tabular}{|c|c|c|c|c|c|c|}
\hline & \multicolumn{2}{|c|}{$R^{2}=832$} & \multicolumn{2}{|c|}{$R^{2}=874$} & \multicolumn{2}{|c|}{$R^{2}=897$} \\
\hline & \multicolumn{2}{|c|}{$\tau=1.1135$} & \multicolumn{2}{|c|}{$\tau=2.42695$} & \multicolumn{2}{|c|}{$\tau=4$} \\
\hline & Max & Min & $\operatorname{Max}$ & Min & $\operatorname{Max}$ & Min \\
\hline$u$ & $4.21 \mathrm{E}-05$ & $-4.02 \mathrm{E}-05$ & $1.09 \mathrm{E}-04$ & $-1.04 \mathrm{E}-04$ & 0.1494 & -0.1437 \\
\hline$v$ & & & & & & \\
\hline$w$ & & & & & & \\
\hline$\xi_{1}$ & & -9.5 & & -2.4 & 35 & -3.322 \\
\hline$\xi_{2}$ & & & & -4.58 & 6.0934 & -6.338 \\
\hline$\xi_{3}$ & & -3.0 & & -6.9 & 0.8836 & -0.882 \\
\hline$\psi_{1}$ & 2.85E-06 & -2.8 & 6 & $-7.13 \mathrm{H}$ & 0.0095 & -0.0095 \\
\hline$\psi_{2}$ & $5.20 \mathrm{E}-06$ & -4.96 & & -1.27 & & -0.0174 \\
\hline$\theta$ & $5.06 \mathrm{E}-06$ & $-2.50 \mathrm{E}$ & $1.26 \mathrm{E}-05$ & $-6.67 \mathrm{E}$ & 0.0169 & -0.0094 \\
\hline$\phi$ & $9.75 \mathrm{E}-06$ & $-5.11 \mathrm{E}-06$ & $2.42 \mathrm{E}-05$ & $-1.33 \mathrm{E}-05$ & 0.0324 & -0.0185 \\
\hline
\end{tabular}

Table 3: Summary of numerical results for $T_{f}=8, \epsilon=0.1, L e=5, \lambda=0.1, R_{c}=10$, $R a_{L}=904.3747, R a_{E}=414.5583, L x=1.4$ and $L y=1$. 


\begin{tabular}{ccccccccc}
\hline & \multicolumn{3}{c}{$R^{2}=240$} & \multicolumn{2}{c}{$R^{2}=258$} & \multicolumn{2}{c}{$R^{2}=276$} & \multicolumn{2}{c}{$R^{2}=292$} \\
\hline & \multicolumn{2}{c}{$\tau=0.79925$} & \multicolumn{2}{c}{$\tau=2.916$} & \multicolumn{2}{c}{$\tau=2.4058$} & \multicolumn{2}{c}{$\tau=2.0641$} \\
\hline & Max & Min & Max & Min & Max & Min & Max & Min \\
\hline$u$ & $2.10 \mathrm{E}-05$ & $-2.13 \mathrm{E}-05$ & 19.18 & -19.18 & 20.77 & -20.77 & 22.11 & -22.11 \\
$v$ & $1.50 \mathrm{E}-05$ & $-1.51 \mathrm{E}-05$ & 18.97 & -18.97 & 20.98 & -20.98 & 22.66 & -22.66 \\
$w$ & $2.53 \mathrm{E}-05$ & $-1.04 \mathrm{E}-05$ & 41.88 & -13.25 & 46.69 & -15.53 & 50.95 & -17.45 \\
$\xi_{1}$ & $7.39 \mathrm{E}-04$ & $-7.36 \mathrm{E}-04$ & 862.68 & -862.68 & 955.33 & -955.33 & 1034.00 & -1034.00 \\
$\xi_{2}$ & $1.01 \mathrm{E}-03$ & $-1.02 \mathrm{E}-03$ & 847.44 & -847.44 & 918.44 & -918.44 & 978.53 & -978.53 \\
$\xi_{3}$ & $1.56 \mathrm{E}-04$ & $-1.53 \mathrm{E}-04$ & 261.03 & -261.03 & 308.62 & -308.62 & 348.50 & -348.50 \\
$\psi_{1}$ & $1.84 \mathrm{E}-06$ & $-1.85 \mathrm{E}-06$ & 3.89 & -3.89 & 4.31 & -4.31 & 4.66 & -4.66 \\
$\psi_{2}$ & $2.71 \mathrm{E}-06$ & $-2.67 \mathrm{E}-06$ & 4.30 & -4.30 & 4.71 & -4.71 & 5.04 & -5.04 \\
$\theta$ & $4.25 \mathrm{E}-06$ & $-1.52 \mathrm{E}-06$ & 4.15 & -0.68 & 4.42 & -0.79 & 4.64 & -0.88 \\
$\phi$ & $1.29 \mathrm{E}-06$ & $-5.50 \mathrm{E}-07$ & 1.56 & -0.23 & 1.60 & -0.25 & 1.63 & -0.28 \\
\hline
\end{tabular}

Table 4: Summary of numerical results for $T_{f}=-11, \epsilon=0.5, L e=5, \lambda=0.1, R_{c}=5$, $R a_{L}=318.1784, R a_{E}=155.5336, L x=1.8$ and $L y=1.2$.

\section{References}

[1] S. K. Jena, S. K. Mahapatra, A. Sarkar, Double diffusive buoyancy opposed natural convection in a porous cavity having partially active vertical walls, Int. J. Heat Mass Transfer 62 (2013) 808-817.

[2] S. K. Jena, S. K. Mahapatra, A. Sarkar, Thermosolutal Convection in a Rectangular Concentric Annulus: A Comprehensive Study, Transp. Porous Med. 98 (2013) 103-124.

[3] B. Chen, A. Cunningham, R. Ewing, R. Peralta, E. Visser, Twodimensional modelling of microscale transport and biotransformation in porous media, Numerical Methods for PDEs 10 (1994) 65-83.

[4] B.J. Suchomel, B.M. Chen, M.B. Allen, Network model of flow, transport and biofilm effects in porous media, Transp. Porous Med. 30 (1998) 1-23.

[5] M.C. Curran, M.B. Allen, Parallel computing for solute transport models via alternating direction collocation. Adv. Water Res. 13 (1990) 70-75.

[6] R.E. Ewing, S. Weekes, Numerical methods for contaminant transport in porous media, Marcel Decker, Inc.: New York, Vol. 202. (1998) 75-95. 
[7] F. Franchi, B. Straughan , A comparison of the GraC and KazhikhovSmagulov models for top heavy pollution instability, Adv. Water Res. 24 (2001) 585-594.

[8] A. Ludvigsen, E. Palm, R. McKibbin , Convective momentum and mass transport in porous sloping layers, J. Geophysical Res. 97 (2001) 1231512325 .

[9] A. Gilman, J. Bear, The influence of free convection on soil salinization in arid regions, Transp. Porous Med. 23 (1996) 275-301.

[10] I.S. Shivakumara, S.P. Suma, Effects of throughflow and internal heat generation on the onset of convection in a fluid layer, Acta Mech. 140 (2000) 207-217.

[11] I.S. Shivakumara, A. Khalili, On the stability of double diffusive convection in a porous layer with throughflow, Acta Mech. 152 (2001) 165-175.

[12] I.S. Shivakumara, A. Khalili, Non-Darcian effects on the onset of convection in a porous layer with throughflow, Transp. Porous Med. 53 (2003) 245-63.

[13] I.S. Shivakumara, S. Sureshkumar, Convective instabilities in a viscoelastic-fluid-saturated porous medium with throughflow, J. Geophys. Eng. 4 (2007) 104-15.

[14] D.A. Nield, A.V. Kuznetsov, The effect of vertical throughflow on thermal instability in a porous medium layer saturated by a Nanofluid, Transp. Porous Med, 87 (2011) 765-775.

[15] A.A. Hill, Unconditional nonlinear stability for convection in a porous medium with vertical throughflow, Acta Mech. 193 (2007) 197-206.

[16] A.A. Hill, S. Rionero, B. Straughan, Global stability for penetrative convection with throughflow in a porous material, IMA J. App. Math. 72 (2007) 635-643.

[17] F. Capone, M. Gentile, A.A. Hill, Penetrative convection in a fluid layer with throughflow Ricerche di Matematica 57 (2008) 251-260. 
[18] I.S. Shivakumara, C.E. Nanjundappa, Effects of quadratic drag and throughflow on double diffusive convection in a porous layer, Int. Commun. Heat Mass Transfer 33 (2006) 357-363.

[19] I.S. Shivakumara, S. Sureshkumar, Effects of throughflow and quadratic drag on the stability of a doubly diffusive Oldroyd-B fluid-saturated porous layer, J. Geophys. Eng. 5 (2008) 268-280.

[20] A.A. Altawallbeh, B.S. Bhadauria, I. Hashim, Linear and nonlinear double-diffusive convection in a saturated anisotropic porous layer with Soret effect and internal heat source, Int. J. Heat Mass Transfer 59 (2013) 103-111.

[21] F. Capone, M. Gentile, A.A. Hill, Double-diffusive penetrative convection simulated via internal heating in an anisotropic porous layer with throughflow, Int. J. Heat Mass Transfer 45 (2011) 1622-1626.

[22] B. Straughan, The energy method, stability, and nonlinear convection, Applied Mathematical Sciences, second ed., vol. 91, Springer, 2004.

[23] D.A. Nield, A. Bejan, Convection in Porous Media, third ed., SpringerVerlag, New York, 2006.

[24] F. Capone, M. Gentile, A.A. Hill, Penetrative convection via internal heating in anisotropic porous media, Mech. Res. Com. 37 (2010) 441444 .

[25] B. Straughan, A.J. Harfash, Instability in Poiseuille flow in a porous medium with slip boundary conditions, Microfluid Nanofluid, 15 (2013) 109-115.

[26] A.J. Harfash, Magnetic effect on instability and nonlinear stability of double diffusive convection in a reacting fluid, Contin. Mech. Thermodyn. 25 (2013) 89-106.

[27] A.J. Harfash, B. Straughan, Magnetic effect on instability and nonlinear stability in a reacting fluid, Meccanica. 47 (2012) 1849-1857.

[28] H. Fasel, Investigation of the stability of boundary layers by a finitedifference model of the Navier-Stokes equations. J. of Fluid Mech., 78 (1976) 355-383. 
[29] M. Napolitano, L. A. Catalano, A multigrid solver for the vorticityvelocity Navier-Stokes equations. Int. J. Numer. Meth. Fluids, 13 (1993) 49-59.

[30] G. Guj and F. Stella, A vorticity-velocity method for the numerical solution of 3D incompressible flows. J. Comput. Phys., 106 (1993) 286298.

[31] C. Davis and P. W. Carpenter, A novel velocity-vorticity formulation of the Navier- Stokes equations with application to boundary layer disturbance evolution. J. Comp. Phys., 172 (2001) 119-165.

[32] K. L. Wong and A. J. Baker, A 3D incompressible Navier-Stokes velocity-vorticity weak form finite element algorithm. Int. J. Numer. Meth. Fluids, 38 (2002) 99-123.

[33] G. D. Mallinson and G. de Vahl Davis, Three-dimensional natural convection in a box: a numerical study. J. Fluid Mech., 83 (1977) 1-31.

[34] O. Daube, Resolution of the 2D Navier-Stokes equations in velocityvorticity form by means of an influence matrix technique. J. Comput. Phys., 103 (1992) 402-414.

[35] G. Guevremont, W. G. Habashi, M. M. Hafez, Finite element solution of the Navier-Stokes equations by a velocity-vorticity method. Int. J. Numer. Meth. Fluids, 10 (1990) 461-475. 

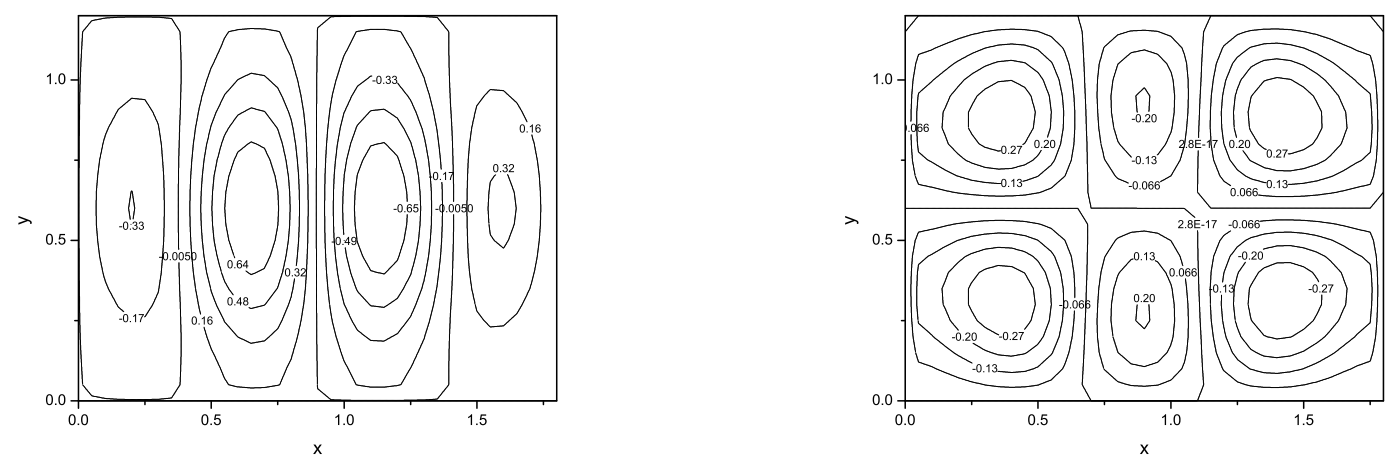

(a)
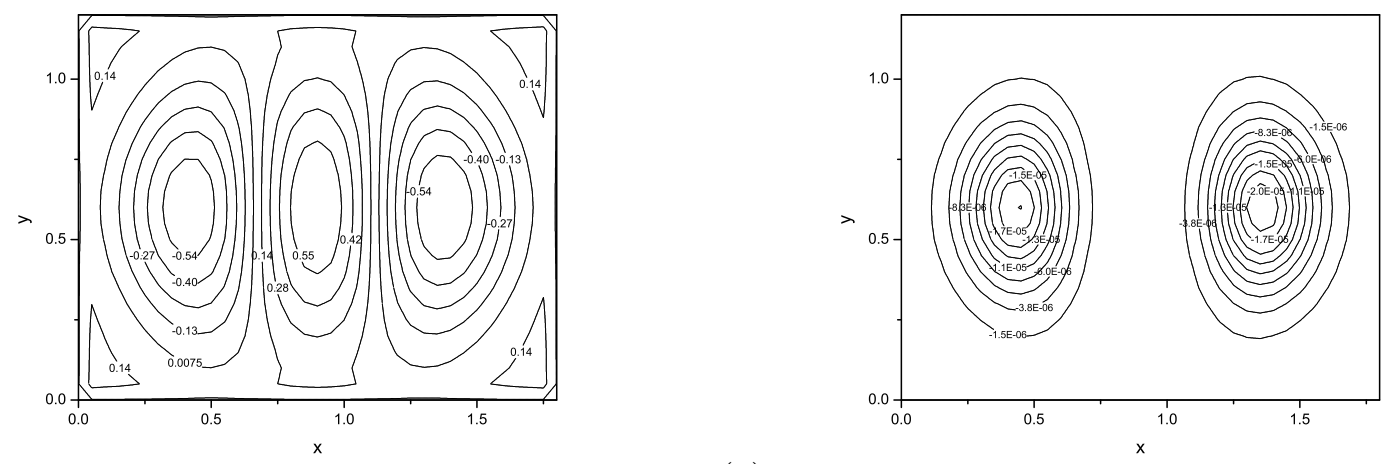

(c)

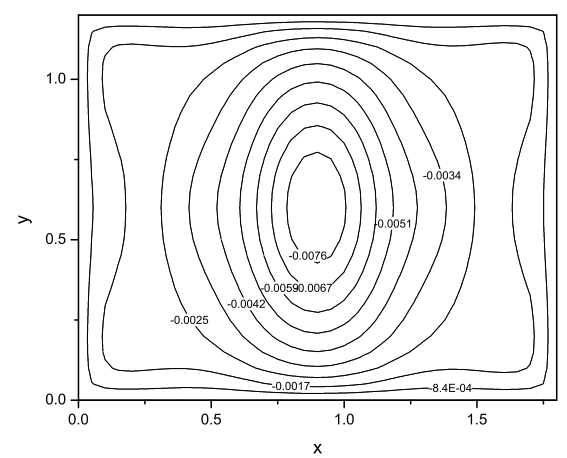

(e)

Figure 2: The contour maps at $z=0.5, L e=5, \lambda=0.1 T_{f}=6, \epsilon=0.1, R_{c}=10$, $L x=1.8, L y=1.2, R^{2}=782, \Delta t=5 \times 10^{-5}, \Delta x=\Delta y=\Delta z=0.02$. (a) $u,(b)$ $v,(c) w,(d) \phi,(e) \theta$. 